\title{
Template-directed self-assembled magnetic nanostructures for probe recording
}

\author{
P. Kappenberger, ${ }^{1, a)}$ F. Luo, ${ }^{2, b)}$ L. J. Heyderman, ${ }^{2}$ H. H. Solak, ${ }^{2}$ C. Padeste, ${ }^{2}$ \\ C. Brombacher, ${ }^{3}$ D. Makarov, ${ }^{3, b)}$ T. V. Ashworth, ${ }^{4,5}$ L. Philippe, ${ }^{6}$ H. J. Hug, ${ }^{1,5}$ and \\ M. Albrecht ${ }^{3}$ \\ ${ }^{1}$ EMPA, Nanoscale Materials Science, CH-8600 Dübendorf, Switzerland \\ ${ }^{2}$ Laboratory for Micro- and Nanotechnology, Paul Scherrer Institut, CH-5232 Villigen PSI, Switzerland \\ ${ }^{3}$ Institute of Physics, Chemnitz University of Technology, D-09107 Chemnitz, Germany \\ ${ }^{4}$ NanoScan Ltd., $\mathrm{CH}-8600$ Dübendorf, Switzerland \\ ${ }^{5}$ Department of Physics, University of Basel, 4056 Basel, Switzerland \\ ${ }^{6}$ EMPA, Laboratory for Mechanics of Materials and Nanostructures, CH-3602 Thun, Switzerland
}

(Received 2 May 2009; accepted 14 June 2009; published online 16 July 2009)

\begin{abstract}
We employ a template-directed self-assembly process to arrange nanospheres as small as $20 \mathrm{~nm}$ in a polymer resist template with regular hole arrays with periods down to $42 \mathrm{~nm}$ produced using extreme ultraviolet interference lithography. To demonstrate magnetic probe recording employing magnetic tips, an array of magnetic caps with perpendicular magnetic anisotropy was then created by depositing $\mathrm{Co} / \mathrm{Pt}$ multilayer films on $60 \mathrm{~nm}$ nanospheres, arranged on a square lattice with $100 \mathrm{~nm}$ period. The magnetic nanocaps can be switched individually in a controlled fashion, with recognition of a successful switching event realized by measurement of a force-distance curve. (C) 2009 American Institute of Physics. [DOI: 10.1063/1.3176937]
\end{abstract}

The capacity of hard disk drives (HDDs) is determined by the physical size of a bit of information, and in conventional HDDs today an individual bit consists of weakly magnetically coupled grains. To further increase the areal storage density, a reduction of the grain size is required to maintain the signal-to-noise ratio. However, this reduction has a lower boundary, the superparamagnetic limit, ${ }^{1}$ at which the magnetic information stored in the media becomes thermally unstable. Therefore, a concept of regular arrays of magnetic nanostructures where an information bit is stored in a single nanostructure, so-called magnetic bit patterned media, was introduced. $^{2}$ However, several challenges have to be addressed: on the one hand, techniques for fabricating ordered arrays of magnetic nanostructures over large areas are needed and, on the other hand, methods need to be found for successful writing of information in the bit patterned media. For probe recording, an atomic force microscopy cantilever tip coated with a magnetic material used to read/write information to each magnetic nanostructure is considered to be promising. ${ }^{3}$ Probe recording has also been achieved in nonmagnetic materials. ${ }^{4,5}$ However, these techniques suffer from the relatively slow reading and writing speed. Therefore, large scale parallelization of the tips is required to boost performance as demonstrated in the Millipede system. ${ }^{3,5}$

In addition to issues with the writing process, considerable efforts have been dedicated to create ordered arrays of magnetic nanostructures. To produce bit patterned media, numerous lithography techniques, including electron beam, $\mathrm{x}$-ray, and nanoimprint lithography, have been applied. ${ }^{6,7}$ Moreover, various template-directed self-assembly approaches such as phase separation in block copolymers ${ }^{8}$ and self-assembled magnetic particles ${ }^{9,10}$ have been intensively investigated. An alternative method, which combines the

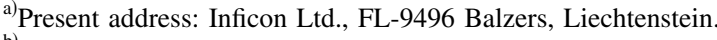

b) Authors to whom correspondence should be addressed. Electronic addresses: denys.makarov@physik.tu-chemnitz.de and feng.luo@psi.ch.
}

self-assembly of nonmagnetic spherical nanoparticles with defined magnetic properties provided by a magnetic film deposited onto the particles, was recently introduced. ${ }^{11}$ However, for this to be implemented as bit patterned media, methods have to be developed to order the nanoparticles over large areas.

In this letter we present a template-directed selfassembly process to arrange nanospheres with sizes as small as $20 \mathrm{~nm}$ on a template consisting of a regular hole array in a polymer resist with a period down to $42 \mathrm{~nm}$ produced using extreme ultraviolet interference lithography (EUV-IL). In order to ascertain the applicability of the assembly of spheres for magnetic recording, an array of magnetic caps with perpendicular magnetic anisotropy was then created by depositing $\mathrm{Co} / \mathrm{Pt}$ multilayers on nanospheres with a diameter of $60 \mathrm{~nm}$ arranged on a square lattice with a period of $100 \mathrm{~nm}$. This sample was then employed to perform a magnetic probe recording study using MFM tips.

EUV-IL at the XIL beamline (Swiss Light Source) is employed to create patterns in the form of arrays of holes in a thin polymethyl methacrylate (PMMA) resist layer on a $\mathrm{Si}$ substrate. ${ }^{12}$ The substrate is then tilted by $15^{\circ}$ and a colloidal solution consisting of sulphatized polystyrene nanospheres (IDC, Portland, OR) diluted with Milli-Q water is dropped onto the substrate so that the nanospheres are trapped in the template holes as the solution flows slowly downwards. As the colloidal solution dries, the nanospheres are fixed inside the holes, and a dense long-range order is formed. A typical patterned hole array in a PMMA resist with a period of $100 \mathrm{~nm}$ on a Si substrate is shown in Fig. 1(a). The hole size is $60 \mathrm{~nm}$ with a resist thickness of $25 \mathrm{~nm}$. Nanospheres with a diameter of $60 \pm 15 \mathrm{~nm}$ which have been captured in the $60 \mathrm{~nm}$ holes to form a perfect regular square lattice are shown in Fig. 1(b). This template-directed ordering method proved to be very tolerant of the dispersion in the nanosphere size, and we were able to achieve long-range ordering of the colloidal nanospheres over areas up to $5 \times 5 \mu \mathrm{m}^{2}$. This dem- 

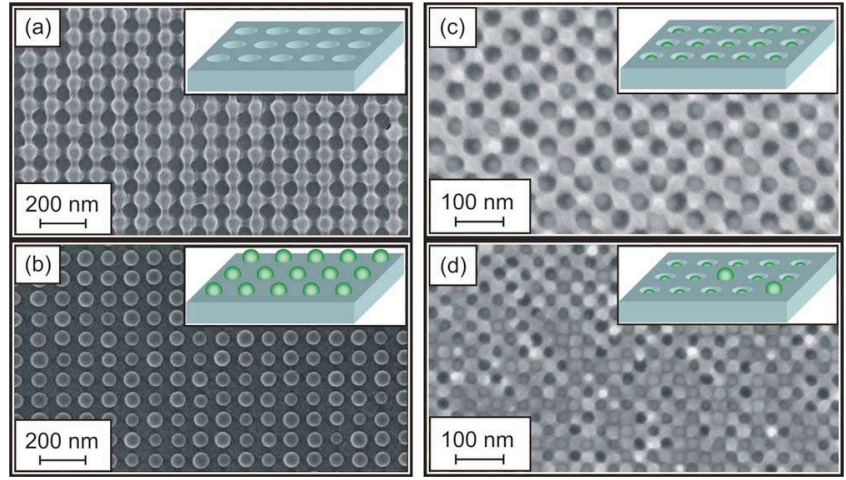

FIG. 1. (Color online) SEM images of (a) a patterned hole array in a PMMA resist created with EUV-IL with a hole size of $60 \mathrm{~nm}$ and a period of $100 \mathrm{~nm}$. (b) Nanospheres with a diameter of $60 \mathrm{~nm}$ captured in the regular square hole array shown in (a). Template-directed self-assembly of $20 \pm 5 \mathrm{~nm}$ nanospheres in (c) template with a hole diameter of $30 \mathrm{~nm}$ and a period of $50 \mathrm{~nm}$ and (d) template with a hole diameter of $22 \mathrm{~nm}$ and a period of $42 \mathrm{~nm}$. The ordering is schematically shown in the insets.

onstrates an improvement compared to the conventional selfassembly scheme, where there is only a close-packed shortrange order of the nanospheres with many defects in an array due in part to the particle size distribution.

We also attempted to apply this template-directed assembly process to order even smaller nanospheres with a diameter of $20 \pm 5 \mathrm{~nm}$ in regular hole arrays with two periods of 50 and $42 \mathrm{~nm}$ and with a hole size of 30 and $22 \mathrm{~nm}$, respectively. The self-assembly on both templates works very well, although not perfectly. For the larger period template $($ period $=50 \mathrm{~nm})$, the nanospheres with a diameter of $20 \mathrm{~nm}$ are smaller than the hole size of $30 \mathrm{~nm}$. Therefore, the nanospheres fall into the holes [Fig. 1(c)]. For the smaller period template (period $=42 \mathrm{~nm}$ ), the hole size is reduced to $22 \mathrm{~nm}$. The smaller spheres again fall into the holes, while the larger spheres sit on top of the holes as presented in Fig. 1(d). For both templates, there are a few empty holes (less than 5\%) and occasionally there are places where the nanospheres are positioned on top of the PMMA resist.

Magnetic caps were produced by deposition of a $[\operatorname{Pt}(0.8 \mathrm{~nm}) / \mathrm{Co}(0.3 \mathrm{~nm})]_{8} / \operatorname{Pt}(5 \mathrm{~nm})$ multilayer stack $^{13}$ on the $60 \mathrm{~nm}$ nanosphere assembly with a period of $100 \mathrm{~nm}$. An additional 1-nm-thick Pt capping layer was deposited in order to prevent oxidation. The smaller periods are not used due to the current challenges associated with addressing nanospheres at smaller dimensions and the sample morphology, i.e., the $20 \mathrm{~nm}$ nanospheres are fully embedded in a hole array. Magnetic characterization was performed using polar magneto-optic Kerr effect magnetometry (MOKE). The hysteresis loop measured on the magnetic film grown on an unpatterned region of the substrate reveals a typical easy axis loop with an out-of-plane coercivity of about $130 \mathrm{mT}$ [Fig. 2(a), filled squares]. In addition, the magnetic properties of the array of magnetic caps were investigated using MFM. An MFM image taken on the sample in the demagnetized state is presented in Fig. 2(b). Dark or bright contrast is visible on each nanocap, indicating that the caps are in a single-domain magnetic state with an averaged magnetization direction pointing perpendicular to the substrate. It should be noted that magnetic contrast also arises from the large magnetic domains in the film surrounding the particles.

To investigate the switching field distribution (SFD) in the array of magnetic nanocaps, a series of MFM images

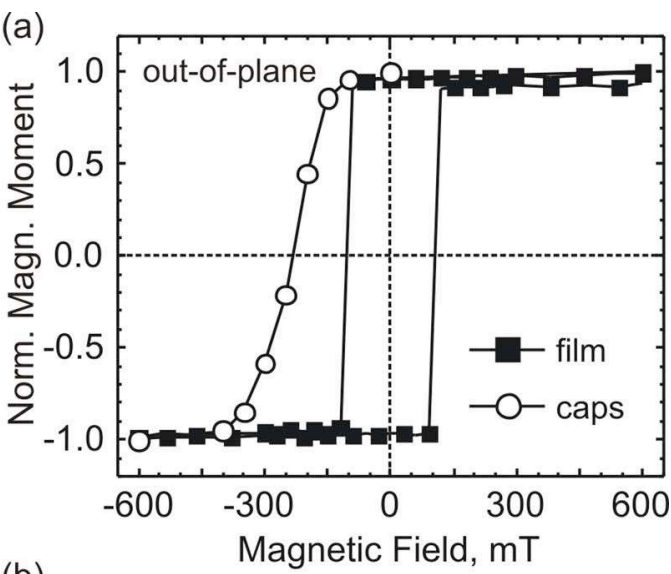

(b)

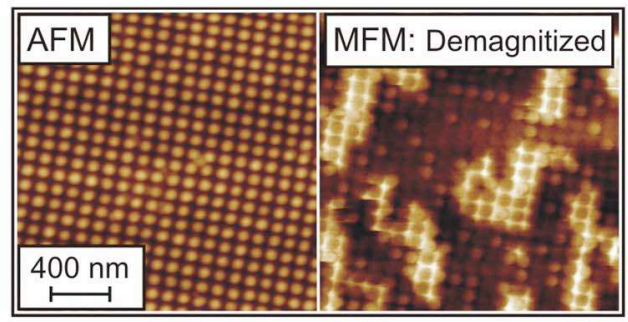

(c)

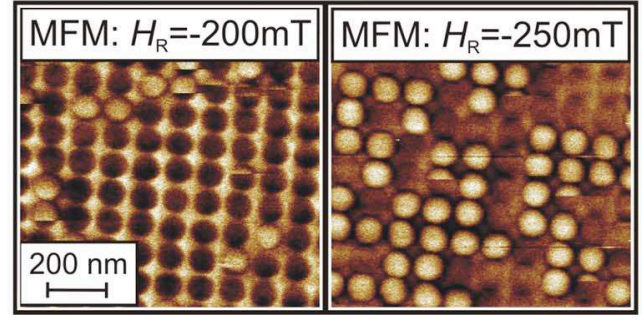

FIG. 2. (Color online) (a) Polar-MOKE hysteresis loop taken on the $\mathrm{Co} / \mathrm{Pt}$ multilayer stack grown on an unpatterned area of the substrate (filled squares). (b) AFM/MFM images of magnetic nanocaps following demagnetization (nanocap diameter: $60 \mathrm{~nm}$, period: $100 \mathrm{~nm}$ ). (c) MFM images taken at remanence after exposing the initially saturated samples to a reverse magnetic field, $H_{R}$. The corresponding remanence curve is constructed by counting the number of reversed nanostructures as a function of applied reverse field [open symbols in (a)].

were taken at remanence after exposing the samples to increasing reverse magnetic field [Fig. 2(c)], after an initial saturation of the sample magnetization. The MFM images were analyzed and a remanence curve was constructed by counting the number of reversed magnetic caps as a function of applied reverse field [Fig. 2(a), open circles], showing an average switching field of about $230 \mathrm{mT}$ with a rather broad SFD as is usually observed in bit patterned media. 7,14

In order to perform probe recording on the prepared array of magnetic nanocaps, the MFM tip has to fulfill two critical requirements: the stray field of the MFM tip has to be sufficiently large to reverse the magnetization direction of the individual magnetic cap and the spatial distribution of the stray field has to be rather narrow so that the magnetic state of the neighboring magnetic nanostructures is not affected. Thus, a careful calibration of the magnetic stray field of the MFM tips ascertains its quality for magnetic recording. ${ }^{15}$ Commercially available precoated MFM cantilevers consist of a magnetic CoCrPt layer with thicknesses between 3 and $25 \mathrm{~nm}$, which covers the tip, the cantilever bar and even parts of the support chip. The stray field of less than $10 \mathrm{mT}$ of these commercial tips is generally too weak for probe record- 


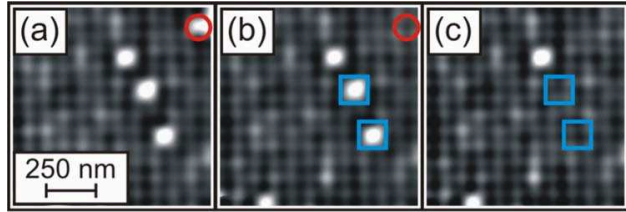

(d)

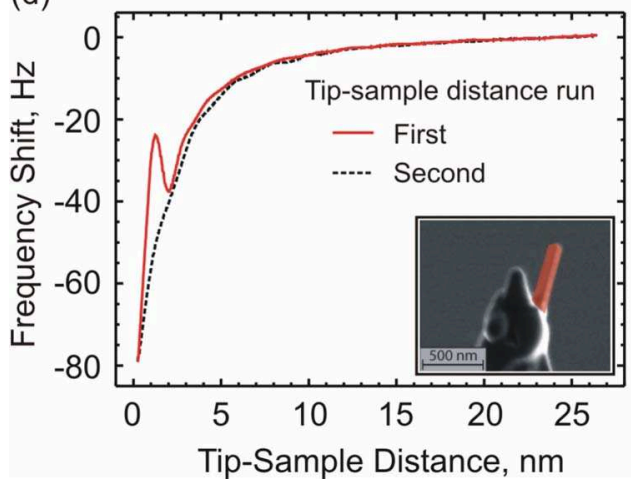

FIG. 3. (Color online) Probe recording on an array of nanocaps on $60 \mathrm{~nm}$ nanospheres with a period of $100 \mathrm{~nm}$. [(a)-(c)] The series of MFM images reveals that the magnetization of the dots indicated with a frame switch with the tip field from an antiparallel (bright) to a parallel configuration (dark). The graph in (d) shows force-distance curves displaying a kink when the magnetization of the nanocap changes its direction. A custom made rodlike tip used for probe recording experiments is shown as an inset in (d).

ing applications. Therefore, in order to increase the tip's stray field, a series of MFM tips was specially manufactured. In order to assess the geometry and strength of the stray field produced by each tip, a standard calibration procedure was applied. ${ }^{15}$ The largest stray field of $67.5 \mathrm{mT}$ was achieved by using a 400-nm-long Co rod with a diameter of $100 \mathrm{~nm}$ grown by electrochemical deposition inside an aluminum membrane and attached near the apex of the cantilever tip. To focus its stray field, the rod's apex was further shaped into a cone by focused ion beam [inset in Fig. 3(d)] resulting in an apex diameter of less than $25 \mathrm{~nm}$.

The proof-of-concept probe recording measurements were performed on a variable temperature UHV-MFM at a base temperature of $8 \mathrm{~K}$. The setup is equipped with a superconducting coil, which provides a homogenous bias field. The MFM data was acquired using the noncontact dynamic mode. The SFD within the scan area of the MFM was mapped. In the actual probe recording experiment, a magnetic bias field was additionally applied in order to overcome the switching threshold of an individual nanocap. However, one has to be aware that the applied bias field will result in a reduction of thermal stability of the entire media.

The magnetic state of the sample in an applied bias magnetic field of $405 \mathrm{mT}$ is presented in Fig. 3(a). In agreement with the magnetic hysteresis loop [Fig. 2(a)], the sample is almost magnetically saturated with only a few magnetic caps left with a reverse magnetization (in white) which we use for the probe recording trials. In order to initiate a magnetic writing event, the MFM tip was positioned over the nanocap of choice. Then the oscillation amplitude was reduced from 10 to $3.5 \mathrm{~nm}$ peak-to-peak and a force-distance curve was acquired by approaching the cantilever slowly in vertical direction [Fig. 3(d), red solid curve]. The spectra shows a wellpronounced peak at a tip-sample separation distance of about $1.2 \mathrm{~nm}$, indicating a magnetic switching event [island in redcircular frame in Fig. 3(a)], which is confirmed by the next MFM scan performed at larger tip-sample separation [Fig. 3(b)]. Moreover, in an additional measurement taken after the switching event at the same location [Fig. 3(d), black dashed curve] no peak is present. Indeed, the presence of the peak in a force-distance curve is useful for tracking successful switching events during magnetic probe recording. In addition two further switching events are indicated by the blue square frames in Figs. 3(b) and 3(c), clearly demonstrating that using the field-assisted probe-recording approach, isolated nanocaps can be switched in a controlled fashion.

In conclusion, we have developed a template-directed self-assembly process to arrange nanospheres as small as $20 \mathrm{~nm}$ in a regular hole array with periods down to $42 \mathrm{~nm}$ created in polymer resist using EUV-IL. This method allows the tuning of the interparticle distance and, with the use of surface functionalization, could also be used to order other nanoparticles. For potential storage applications beyond $1 \mathrm{Tbit} / \mathrm{in}^{2}$, patterns with periods below $25 \mathrm{~nm}$ are necessary. In order to achieve this, the EUV-IL and self-assembly of the nanospheres will require further development to decrease the template hole period and to obtain a perfect long range order of the nanospheres at these smaller dimensions. An array of magnetic caps was then created by depositing $\mathrm{Co} / \mathrm{Pt}$ multilayers on nanospheres with a diameter of $60 \mathrm{~nm}$ arranged on a square lattice with a period of $100 \mathrm{~nm}$ given by the EUV-IL hole template. A magnetic-writing event was initiated by approaching a MFM tip to the nanocap of choice in the presence of a homogeneous magnetic bias field while acquiring a force-distance curve. This event was detected as a distinct feature in the approach curve. This recognition method can therefore be implemented to track the writing process, providing an essential tool for future magnetic probe recording.

The financial support provided by the European Commission via the STREP project MAFIN (Grant No. 026513) is gratefully acknowledged. Part of this work was performed at the Swiss Light Source, Paul Scherrer Institut, Villigen, Switzerland.

${ }^{1}$ D. Weller and A. Moser, IEEE Trans. Magn. 35, 4423 (1999).

${ }^{2}$ C. A. Ross, Annu. Rev. Mater. Sci. 31, 203 (2001).

${ }^{3}$ H. J. Mamin, B. D. Terris, L. S. Fan, S. Hoen, R. C. Barrett, and D. Rugar, IBM J. Res. Dev. 39, 681 (1995).

${ }^{4}$ E. Gidon, O. Lemonnier, B. Rolland, O. Bichet, and C. Dessler, Appl. Phys. Lett. 85, 6392 (2004).

${ }^{5}$ P. Vettiger, G. Cross, M. Despont, U. Drechsler, U. Dürig, B. Gotsmann, W. Häberle, M. A. Lantz, H. E. Rothuizen, S. Stutz, and G. K. Binning, IEEE Trans. Nanotechnol. 1, 39 (2002).

${ }^{6}$ S. Y. Chou, Proc. IEEE 85, 652 (1997).

${ }^{7}$ F. Luo, L. J. Heyderman, H. H. Solak, T. Thomson, and M. E. Best, Appl. Phys. Lett. 92, 102505 (2008).

${ }^{8}$ I. Bita, J. K. W. Yang, J. S. Jung, C. A. Ross, E. L. Thomas, and K. K. Berggren, Science 321, 939 (2008).

${ }^{9}$ C. L. Cheung, R. J. Nikolić, C. E. Reinhardt, and T. F. Wang, Nanotechnology 17, 1339 (2006).

${ }^{10}$ S. Sun, C. B. Murray, D. Weller, L. Folks, and A. Moser, Science 287, 1989 (2000)

${ }^{11}$ M. Albrecht, G. Hu, I. L. Guhr, T. C. Ulbrich, J. Boneberg, P. Leiderer, and G. Schatz, Nature Mater. 4, 203 (2005).

${ }^{12}$ H. H. Solak, J. Phys. D 39, R171 (2006).

${ }^{13}$ T. C. Ulbrich, D. Assmann, and M. Albrecht, J. Appl. Phys. 104, 084311 (2008).

${ }^{14}$ T. Thomson, G. Hu, and B. D. Terris, Phys. Rev. Lett. 96, 257204 (2006).

${ }^{15}$ P. A. van Schendel, H. J. Hug, and B. Stiefel, J. Appl. Phys. 88, 435 (2000). 